\section{Validation of remote mapping of cochlear implants}

\author{
Robert H Eikelboom 1,2,3, Dona MP Jayakody ${ }^{1,2}$, De Wet \\ Swanepoel $^{1,2,3}$, Samuel Chang ${ }^{1,2}$ and Marcus D Atlas ${ }^{1,2}$
}

Journal of Telemedicine and Telecare 0 (0) $1-7$

(C) The Author(s) 2014

Reprints and permissions: sagepub.co.uk/journalsPermissions.nav DOI: $10.1177 / 1357633 \times 14529234$ jtt.sagepub.com

\begin{abstract}
Summary
Recipients of cochlear implants need to attend regular mapping sessions to adjust their speech processors. We developed a PCbased system for remote mapping, which employed voice, video and text communication. Eleven implant recipients had their implant electrodes programmed (mapped) both by conventional methods and remotely. Speech tests, conducted by a family member or research assistant, were used to test the outcome of the programming during the remote consultation. The recipient and audiologist were surveyed regarding aspects of the teleconsultation. There were no significant differences between conventionally and remotely programmed electrode settings. The speech test results were perfect in most cases. The average time to complete the conventional mapping session was $37 \mathrm{~min}$ and the average time for the remote mapping session was $42 \mathrm{~min}(P=0.034)$. Recipients and the audiologist reported favourably on most aspects of the consultations. Lack of synchronisation between voice and video was the most common problem, which disrupted communication. The assistant was important to overcome this problem. All but one participant indicated they would be willing to use tele-mapping in the future. Remote programming of cochlear implants can be conducted reliably with relatively simple equipment, potentially in the homes of remotely located patients assisted by a family member.
\end{abstract}

Accepted: 18 February 2014

\section{Introduction}

Hearing loss is a very common disability, and more than 360 million people in the world are affected by disabling hearing loss. ${ }^{1}$ Providing hearing health services to the large number of people with a hearing loss is a challenge. Many people in the world have poor access to hearing health specialists, or none at all. ${ }^{2}$ Telehealth is a method for improving hearing health services for people in underserved areas who face long distance travelling to regional or city centres, as well as disruption to family and work when services are not available locally. ${ }^{3}$ Telehealth has been used to deliver newborn hearing screening, screening audiology services to children, hearing assessment of adults, hearing aid fitting, and diagnosis and management of ear disease. ${ }^{4,5}$

The barriers to accessing audiology services also apply for people with a cochlear implant. A cochlear implant is an implantable prosthetic device for people with moderately-severe to profound hearing loss. The implant directly stimulates the auditory nerve via a set of electrodes placed in the cochlea. An external unit mounted behind the ear collects and processes sound, and passes this by electro-magnetic induction through the skin to an adjacent receiver-stimulator placed in a sub-periosteal pocket of the temporal bone, which then converts the signal into a set of electrical impulses and passes them through the electrodes. The device is routinely programmed in a fitting technique, producing what is known as a map.

Best practice protocols for the management of cochlear implant recipients state that they should be assessed at 3, 6, 12 and 24 months after their implant, and thereafter annually. These reviews normally include a check of the maps, the settings of each electrode of the implant to provide optimum hearing and comfort, and some tests of the outcomes. These reviews are necessary due to device (electronic) drifts and changes in the ear of the patient (e.g. ageing). Improvements in sound processing techniques can be applied, and rehabilitation and upgrading of devices may also be discussed.

Early reports of remote mapping of cochlear implants were limited to general descriptions. ${ }^{6}$ The implant audiologist used remote desktop software (software that is able to take control of another computer on a computer network) and videoconferencing equipment, and utilised

\footnotetext{
'Ear Sciences Centre, School of Surgery, The University of Western Australia, Nedlands, Australia

${ }^{2}$ Ear Science Institute Australia, Subiaco, Australia

${ }^{3}$ Department of Speech-Language Pathology and Audiology, University of Pretoria, South Africa
}

Corresponding author:

Professor RH Eikelboom, Suite I Level 2, I Salvado Road, Subiaco, WA 6008, Australia.

Email: rob.eikelboom@earscience.org.au 
another audiologist who was with the cochlear implant recipient. Despite time lags and occasional communication breakdowns, and indications that remote programming might be more costly and take more time, clinicians and patients reported positive attitudes towards this mode of service delivery.

This general model of providing remote mapping services has since been reported in four publications. Ramos et al. ${ }^{7}$ found no significant differences between implant processor levels for electrodes obtained remotely and those obtained in a standard programming session, and there were generally positive responses to remote programming from all participants. McElveen et al. ${ }^{8}$ compared audiological outcomes in a group of patients who were programmed remotely with another group programmed face-to-face in the standard way, and found no significant differences. Wesarg et al. ${ }^{9}$ found no significant difference in current levels of the electrodes related to modes of programming, but the testing centre and order of programming did have a significant effect. Finally, Hughes et al. ${ }^{10}$ found that electrode levels were not significantly different after local and remote programming, and most patients indicated that they would be willing to use telehealth at least some of the time. However, speech perception was significantly poorer in the remote sessions, which was attributed to the remote assessment of speech perception in a non-sound treated environment.

In each of these reports, the system involved remotely controlling a cochlear implant programming computer over a secure network connection. In each case, an audiologist or technician was present with the patient to set up the system and assist in the consultation. The systems were designed for use in clinical settings, where access to supporting clinicians was available. A better system would be one that was turn-key in operation, where the patient or a family member could connect their sound processor to an interface box.

\section{Tele-mapping system}

We designed a tele-mapping system to meet the following requirements: (1) a user-friendly interface that required minimum installation for the cochlear implant patient, (2) provide two-way voice, video and text communication, (3) allow the mapping software at the clinic to control the programming box located with cochlear implant recipient, and (4) to use off-the-shelf peripherals.

The system consisted of two computers, one for the cochlear implant recipient and the other for the audiologist. The latter computer also acted as the communication server.

The cochlear implant recipient computer was a Dell Vostro 220 s (Dell Inc, Round Rock, Texas, USA), with a Dell E2310HC Widescreen Flat Panel computer display (Dell Inc, Round Rock, Texas, USA), a mouse and a keyboard connected via USB ports, and a standard pair of speakers connected to the audio-output of the computer. A Creative HD1080 video camera and microphone
(Creative Technology Ltd, Jurong East, Singapore) was connected to the computer via a USB port. Also connected to the computer was a Digital Interface Box (MED-EL Elektromedizinische GmbH, Innsbruck, Austria) which provided a connection to the cochlear implant. This device was connected to the computer via a serial RS232 port.

The audiologist's computer was also a Dell Vostro $220 \mathrm{~s}$, with a $58 \mathrm{~cm}$ LCD computer display, and a mouse and a keyboard connected via USB ports. A Logitech QuickCam Sphere AF video camera (Logitech International SA, Morges, Switzerland) was connected to the computer via a USB port. A Sennheisser PC360 headset with microphone (Sennheiser electronic $\mathrm{GmbH}$ \& Co KG, Wenemark, Germany) was available for voice communication.

The software was developed using Visual Studio Express $\mathrm{v} 10.0$ and the .NET framework, and delivered as a Silverlight application (Microsoft Corporation, Redmond, California USA). Silverlight applications are sent to the clients (users that connect to the system via their Internet browser) each time a session is started. The function of the server was to provide the Silverlight service for the users' computers and to manage the users connecting to the system, so that the audiologist could select which recipient to communicate with. The operating systems used were Microsoft Windows 7 on the audiologist's computer, and Windows XP on the recipient's computer.

Advanced Virtual COM Port (KernelPro Software, Vancouver, Canada) was installed on both computers. This utility intercepted COM port data on both computers, transmitted these data across the network connection using TCP/IP, and output these to the specified COM port. Configuration of the software required entering the IP address of the recipient computer.

Maestro 4.1.1 (MED-EL Elektromedizinische GmbH, Innsbruck, Austria) was used for programming the cochlear implant; this was installed only on the audiologist's computer.

The Silverlight service in the browser included the following:

1. A log-in step, which required the user to identify themselves by name if they were a recipient or by name and password if they were an audiologist.

2. For the audiologist, a list of recipients logged-in, and the ability to connect to or disconnect from a listed user.

3. For all users, a panel that included a large image from the video-camera of the other user, a smaller image of the user at that computer, a box for entering text messages for the other user, another text box that showed the text messages from both users, and an array of response buttons that could be activated by the recipient to indicate the comfort level of stimuli presented by the audiologist (Figure 1).

4. For all users, a panel that enabled the setting of the video frame rate, image size and compression level, 


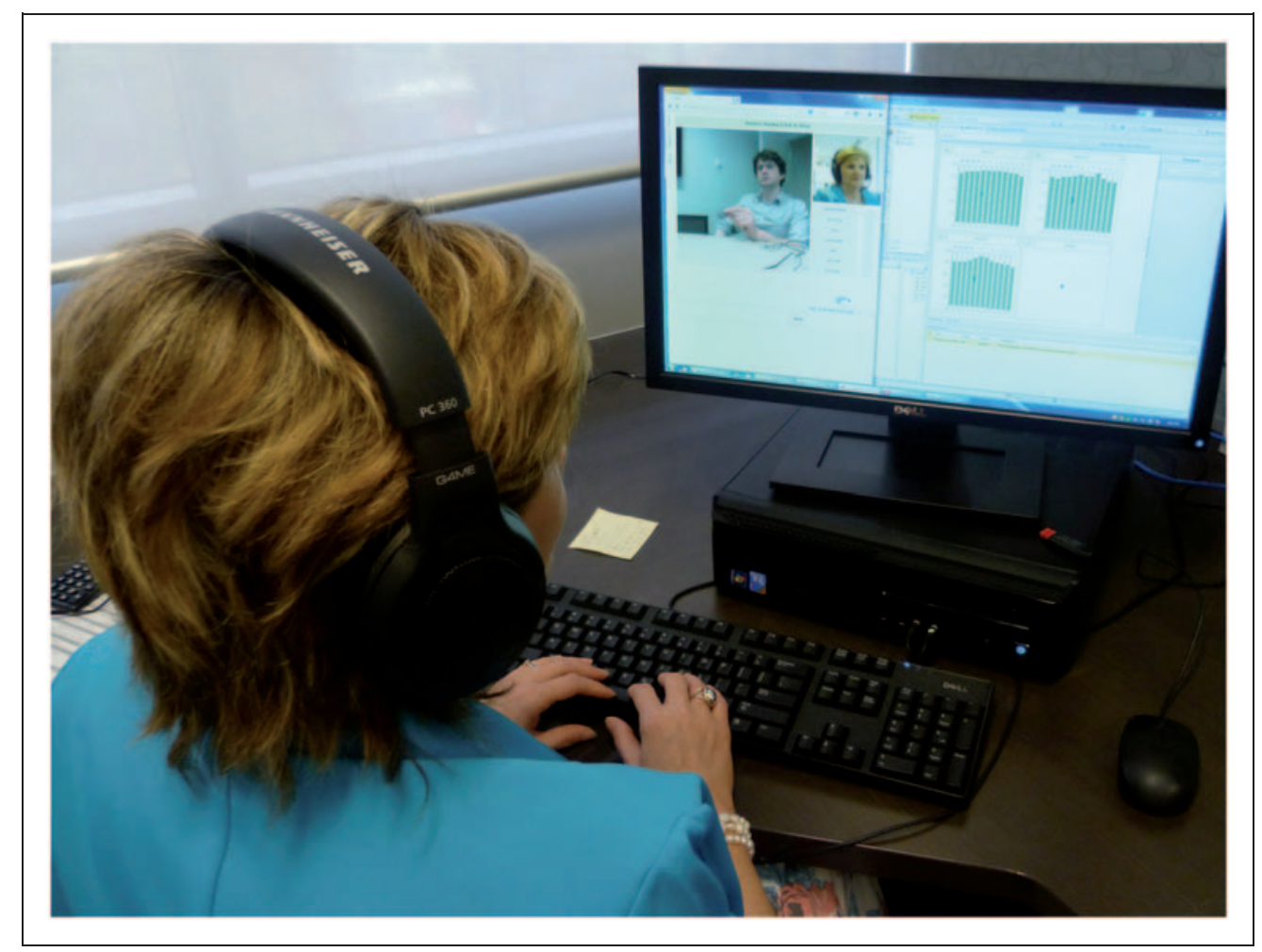

Figure I. Tele-mapping system in use by the audiologist.

and voice sampling rate, bits per sample, channels and frame size. There was also an option for video to be disabled in cases of low bandwidth so that preference could be given for voice communication.

The system connected the computers via a Virtual Private Network (VPN). This set up a private and secure connection across the Internet.

During the development of the system various combinations of voice and video compression were tested. Special coding was used to synchronise the voice and video information. The appropriate settings were determined which produced the best voice quality, with the voice being synchronous with the video, and satisfactory video quality. The video settings were: image size: 320 x 180 pixels; frame rate: 15 frames/second; compression $60 \%$. The sound settings were: sampling rate $22 \mathrm{kbit} / \mathrm{s}$ at $16 \mathrm{bits} / \mathrm{sample}$, one channel (i.e. not stereo) and an audio frame size of 300 . The software was trialled with one cochlear implant recipient before conducting the study.

The aim of the present study was to evaluate our telemapping system for the remote programming of cochlear implants in the home of recipients.

\section{Methods}

Invitation letters or email messages were sent to adult cochlear implant recipients at the Ear Science Institute Australia's Implant Clinic. The inclusion criteria were: a Med-el hearing implant with a fully active electrode; living a short distance $(<20 \mathrm{~km})$ from the clinic or coming into the clinic for an annual appointment. The exclusion criteria were: known neurological or physical handicaps that would have prevented the subject from completing the tasks. The study was approved by the appropriate ethics committee.

\section{Testing procedure}

Mapping was conducted twice, in a conventional faceto-face manner with an implant audiologist, and remotely by the same audiologist. The same audiologist was used to minimise inter-audiologist variation. Half of the participants completed the conventional programming session first before remote programming and the remaining participants completed the remote programming session first.

The remote mapping was conducted in another room in the same building. The network connection between the computers was via a local area network with a bandwidth of $1 \mathrm{Gbit} / \mathrm{s}$.

During the remote mapping session the cochlear implant recipient was assisted by a facilitator (a research assistant or a family member). The facilitators had no skills in cochlear implant patient management, and followed instructions from the audiologist to administer subjective outcomes tests, and repeat instructions in case the recipient did not understand the verbal instructions.

Both types of mapping were completed according to the standard protocols. Electrode impedances and short/ open circuits were determined, maximum comfort levels (MCL) were determined for all active electrodes, and then 
loudness balancing undertaken. For the purpose of the study, threshold levels were set at $10 \%$ of the most comfortable level.

Outcomes were tested by listening to the facilitator to determine sound level and quality, and by patients listening to the sound of their own voice by counting aloud from 1 to 10. The map was adjusted according to the standard protocols if necessary.

The Ling six sound test ${ }^{11}$ and five open-set sentences (the ability to understand sentences without lip-reading) were administered by the facilitator, and the scores were recorded. Both the Ling six sound test and open-set questions were repeated if the client did not obtain $100 \%$ scores at the first attempt.

After both sessions were completed, the recipient was presented with both cochlear implant maps in turn and asked to comment on the preferred map, based on sound quality, overall loudness and comfort. Finally, the implant map was reset to the one in place before the start of the session. The audiologist recorded the length of time required for both programming sessions.

\section{Survey}

The participant and the audiologist both completed a short questionnaire about the quality of video and voice communication, the ease of communication and their overall experience. Each question was answered on a 5 -point Likert scale. They were also asked whether they would be prepared to use remote mapping for future consultations, and whether they would recommend remote mapping for other people with a cochlear implant (yes or no answers).

\section{Analysis}

All study data were collated on a spreadsheet and statistical analysis was performed with a standard package
(SPSS v20). The MCLs between sessions were analysed by univariate analysis of variance.

The Ling six and open-sentence test results for the two modes of programming were analysed with the Chi-squared test with a Yates correction to determine if they were significantly different. Because of the low numbers, a $2 \times 2$ matrix was formed with one condition being the number of perfect scores, and the other condition being the number of non-perfect scores.

The time taken for the conventional and remote session was analysed with descriptive statistics, and compared to the binomial variables age, gender, order of testing and number of implants.

The Likert scores were treated as continuous variables, ${ }^{12}$ and for each question the scores for audiologist and participant was compared by univariate analysis of variance.

\section{Results}

Eleven people participated in the study (Table 1). One other person was excluded because she had a neurological condition that would have prevented her from providing reliable responses during the programming. Five of the 11 participants were men. The mean age was 68 years (range 57-77). Seven of the participants had one cochlear implant, and all but one had a contralateral hearing aid; the other four had bilateral implants. The implants of five right ears and six left ears were tested. The mean duration of deafness was 37 years (range 16-57). The mean length of use of the implant was 2.7 years (range 0.36-5.1). Three different types of implants were included in the study, but all participants had the same implant speech processor (Opus2).

\section{Outcomes}

MCL levels: analysis of variance showed that there was no significant difference in MCL due to method.

Table I. Demographic and implant information. All patients used an Opus2 speech processor.

\begin{tabular}{|c|c|c|c|c|c|c|c|}
\hline No & Sex & $\begin{array}{l}\text { Age } \\
\text { (years) }\end{array}$ & Implant type & $\begin{array}{l}\text { Duration } \\
\text { of deafness } \\
\text { (years) }\end{array}$ & $\begin{array}{l}\text { Length of time } \\
\text { with implant } \\
\text { tested (years) }\end{array}$ & $\begin{array}{l}\text { Ear of } \\
\text { implant } \\
\text { tested }\end{array}$ & $\begin{array}{l}\text { Cochlear } \\
\text { implant }\end{array}$ \\
\hline I & Male & 67 & Sonata $_{\mathrm{TI}}{ }^{100}$ & 32 & 2.5 & Right & Unilateral \\
\hline 2 & Female & 71 & Concerto & 56 & 0.4 & Left & Unilateral \\
\hline 3 & Female & 63 & Sonata $_{T 1}{ }^{100}$ & 24 & 4.3 & Left & Bilateral \\
\hline 4 & Male & 76 & Concerto & 39 & 2.4 & Left & Bilateral \\
\hline 5 & Female & 72 & Sonata $_{\mathrm{TI}} 100$ & 20 & 4.8 & Left & Unilateral \\
\hline 6 & Female & 72 & Concerto & 20 & 0.7 & Left & Unilateral \\
\hline 7 & Female & 63 & Sonata $_{\mathrm{TI}}{ }^{100}$ & 38 & 5.1 & Left & Bilateral \\
\hline 8 & Male & 77 & Sonata $_{T I}{ }^{100}$ & 16 & 4.8 & Right & Bilateral \\
\hline 9 & Male & 64 & Sonata $_{T 1}{ }^{100}$ & 57 & 3.2 & Right & Unilateral \\
\hline 10 & Male & 64 & Concerto & 53 & 0.5 & Right & Unilateral \\
\hline II & Male & 57 & Concerto & 56 & 0.9 & Right & Unilateral \\
\hline
\end{tabular}


However, there was a significant electrode effect $(P=0.004)$. Post hoc tests showed that the greatest variation in MCL levels due to method was for the central electrodes, although the differences were not significant.

Ling six test: all 11 patients scored 6 out of 6 for the Ling six test after the remote mapping session, whilst eight scored 6 out of 6 after the conventional mapping session (Table 2). However, these outcomes were not significantly different.

Open-set questions: ten patients answered all five openset questions after the remote mapping session, compared to nine after the conventional mapping session (Table 2). These outcomes were not significantly different.

Map preference: six patients reported no preference between the maps, two preferred the map obtained by the conventional method, and three preferred the map obtained in the remote session.

Time: the average time to complete the conventional mapping session was $36.5 \mathrm{~min}$ (range 30-45), and the average time for the remote mapping session was $41.5 \mathrm{~min}$ (range 30-50). An analysis of variance showed that the difference was significant $(P=0.034)$. No significant relationship of time difference was found with age, gender or order of mapping, uni- or bilateral implants.

\section{Survey responses}

Patients and the audiologist reported that problems did not occur or were rare (Table 3 ). The most frequent problems reported by the patients were that the clinician's face was not properly framed and that the voice of the clinician was broken or absent.

Table 2. Results from the Ling six and the open-set questions tests. The values indicate the number of participants with each score.

\begin{tabular}{ccc}
\hline Ling six score & $\begin{array}{c}\text { Conventional } \\
\text { mapping }\end{array}$ & $\begin{array}{c}\text { Remote } \\
\text { mapping }\end{array}$ \\
\hline 6 & 8 & $1 \mathrm{I}$ \\
5 & 2 & 0 \\
4 & 0 & 0 \\
3 & 1 & 0 \\
2 & 0 & 0 \\
1 & 0 & 0 \\
0 & 0 & 0 \\
\hline Open-set & Conventional & Remote \\
questions & mapping & mapping \\
\hline 5 & 9 & 10 \\
4 & 2 & 1 \\
3 & 0 & 0 \\
2 & 0 & 0 \\
I & 0 & 0 \\
0 & 0 & 0 \\
\hline
\end{tabular}

Analysis of variance of each question, with the score as the dependent variable and the respondent (patient or audiologist) as the nominal independent variable, showed that there were no significant between-subject effects.

In many cases accompanying comments by the patients indicated that the sound and video image of the audiologist was usually unsynchronised, and that the voice of the audiologist was difficult to understand. There were no negative comments about the video image.

Table 3a. Patient (P) and Audiologist (A) assessment of aspects of voice communication. Values shown are the percentages of the responses $(n=I I)$.

\begin{tabular}{lccccc}
\hline & Not at all & Rarely & Sometimes & Often & Always \\
\hline Breakup (P) & 46 & 9 & 27 & 9 & 9 \\
Breakup (A) & 73 & 9 & 0 & 18 & 0 \\
No sound (P) & 64 & 9 & 27 & 0 & 0 \\
No sound (A) & 91 & 0 & 9 & 0 & 0 \\
Background noise (P) & 82 & 9 & 0 & 9 & 0 \\
Background noise (A) & 100 & 0 & 0 & 0 & 0 \\
Too loud (P) & 73 & 9 & 18 & 0 & 0 \\
Too loud (A) & 100 & 0 & 0 & 0 & 0 \\
Too soft (P) & 82 & 9 & 9 & 0 & 0 \\
Too soft (A) & 91 & 0 & 9 & 0 & 0 \\
Video, no sound (P) & 91 & 0 & 9 & 0 & 0 \\
Video, no sound (A) & 100 & 0 & 0 & 0 & 0 \\
\hline
\end{tabular}

Table 3b. Patient $(\mathrm{P})$ and Audiologist $(\mathrm{A})$ assessment of aspects of video communication. Values shown are the percentages of the responses $(\mathrm{n}=\mathrm{II})$.

\begin{tabular}{lccccc}
\hline & Not at all & Rarely & Sometimes & Often & Always \\
\hline Image froze (P) & 82 & 9 & 0 & 9 & 0 \\
Image froze (A) & 73 & 18 & 9 & 0 & 0 \\
Image breakup (P) & 91 & 0 & 0 & 9 & 0 \\
Image breakup (A) & 100 & 0 & 0 & 0 & 0 \\
Image jerky (P) & 82 & 0 & 9 & 9 & 0 \\
Image jerky (A) & 100 & 0 & 0 & 0 & 0 \\
Out of focus (P) & 91 & 0 & 0 & 9 & 0 \\
Out of focus (A) & 82 & 18 & 0 & 0 & 0 \\
Face missing (P) & 73 & 0 & 9 & 9 & 9 \\
Face missing (A) & 100 & 0 & 0 & 0 & 0 \\
Unusual colour (P) & 91 & 9 & 0 & 0 & 0 \\
Unusual colour (A) & 100 & 0 & 0 & 0 & 0 \\
Sound, no video (P) & 100 & 0 & 0 & 0 & 0 \\
Sound, no video (A) & 82 & 18 & 0 & 0 & 0 \\
Image too small (P) & 100 & 0 & 0 & 0 & 0 \\
Image too small (A) & 100 & 0 & 0 & 0 & 0
\end{tabular}


Table 4. Patient $(P)$ and Audiologist $(A)$ assessments of the experience of tele-mapping. Values shown are the percentages of the responses $(n=I I)$.

\begin{tabular}{|c|c|c|c|c|c|}
\hline & $\begin{array}{l}\text { Very } \\
\text { poor }\end{array}$ & Poor & $\begin{array}{l}\text { Neither } \\
\text { poor } \\
\text { or good }\end{array}$ & Good & $\begin{array}{l}\text { Very } \\
\text { good }\end{array}$ \\
\hline Voice experience $(P)$ & 9 & 45 & 27 & 0 & 18 \\
\hline Voice experience $(A)$ & 9 & 9 & 27 & 55 & 0 \\
\hline Video experience $(\mathrm{P})$ & 0 & 0 & 27 & 55 & 18 \\
\hline Video experience $(A)$ & 0 & 0 & 0 & 91 & 9 \\
\hline Overall experience $(\mathrm{P})$ & 0 & 0 & 9 & 45 & 45 \\
\hline Overall experience $(A)$ & 0 & 0 & 9 & 82 & 9 \\
\hline
\end{tabular}

The audiologist also commented that the video and sound signals were not synchronous, and that the sound clarity was not optimum.

When asked for general assessments of the video, sound and of the consultation, the sound was judged by the patients and audiologist to be neither poor nor good, although it was graded slightly better by the audiologist (Table 4). Regarding the video and an overall impression of the consultations, the reports averaged good. Analysis of variance of these three questions showed no significant difference between patients and audiologist.

All but one of the patients indicated that they would be willing to use a remote mapping service in the future, and all of them indicated that they would recommend it for others. Accompanying comments included the need in teleconsultations to have someone with them to assist with communication with the audiologist.

\section{Discussion}

The main consideration in adopting remote programming for cochlear implant recipients is that the outcomes for the recipients are the same as in face-to-face programming. ${ }^{13}$ Secondary considerations are the time taken for the consultation, the quality of the consultation and the performance of the technology, and the attitude of both recipients and clinicians to a remote programming session.

In the present study of 11 cochlear implant recipients, we found no significant difference in the MCLs of electrodes between those determined in a conventional consultation and those determined in a remote consultation. The larger variations found in the central electrodes compared to the apical electrodes, although not significant, can be attributed to the higher dynamic range and higher MCLs in these electrodes. However, mapping a cochlear implant is not a diagnostic procedure, but a fitting and fine tuning method for hearing aids. Therefore, the concept of acceptable levels of variation in MCLs for electrodes is not valid. On the other hand, the fact that the MCLs were not significantly different is a useful finding, and confirms the findings of others. ${ }^{7,9}$

More valuable are the findings that participants performed equally well in the Ling six and open-sentence tests. Although the differences were not significant, slightly better outcomes were achieved with the remote mapping session. This may be related to the different voices used for the two modes (female audiologist during conventional sessions, and in all but one case a male assisting in the remote sessions). These tests for speech sounds are normally used to decide whether the implant has been properly fitted. Future work will be required to validate speech perception tests that are used to determine candidacy, and to compare pre- and postoperative outcomes. ${ }^{10}$

There was no obvious preference for the map resulting from the remote procedure or the face-to-face session. In most cases they were judged to be the same. This outcome measure has not been reported by others. These findings show that remote programming does not produce a different outcome for the patient to that obtained in a conventional mapping session.

Remote consultations took approximately 5 min longer than conventional programming, which is a little more than reported by others. ${ }^{7,9}$ This extra time will have to be allowed for when managing a clinic. ${ }^{14}$ On the other hand, there would be a substantial time saving for recipients. ${ }^{15}$

The patients and audiologist reported few technical problems in either the video or voice. However, in most cases the patients reported that the voice and video were not synchronised, which made lip reading very difficult. The overall rating by the patients of the voice was between poor and neutral. The audiologist also frequently noted that there was a delay in the presentation of the stimulus to the implant and also in receiving responses, requiring an adaptation from standard practices. This problem has been reported previously. ${ }^{7}$ Despite these problems, the patients and audiologist graded the quality of the consultation as good. We observed that the video became an adjunct to the consultation, being more of a reminder of the personal nature of the consultation. On the other hand, the fact that a number of patients noted that the audiologist was not always properly in the video frame is a reminder that the personal aspects of a consultation are important.

The presence of a facilitator assisting the cochlear implant patient was important in the present study. Achieving high quality voice transmission in videoconferencing can often be difficult. Hearing implants and hearing aids do not completely restore hearing, and even with well fitted hearing aids or implants there may be deficits in speech discrimination. ${ }^{16,17}$ As already noted, in our study there was poor synchronisation between voice and video, but despite this the audiologist and the facilitator were able to communicate without any problems. Furthermore, the facilitator was easily able to administer the spoken voice tests after the electrode programming.

The use of a facilitator could be considered to be a disadvantage, and might make the patient dependent on another person for the consultation to take place. However, in most cases patients had a family member or 
friend to fill this role. An alternative may be that prerecorded high quality video clips of special instructions could be played on request by the audiologist.

In future, the inclusion of self-mapping technologies which are being developed for hearing aids ${ }^{18,19}$ may also change the way that hearing implants are programmed.

Finally, there was a very high level of acceptance of remote mapping by the urban-based patients. Although they did not face the challenges of long distance travel to the clinic, in all but one case they were receptive to future remote mapping sessions.

Further work is needed to establish how problems such as poor network bandwidth and differences between personal computers will affect the quality of consultations and the reliability of the software. In cases where the distance between the audiologist and patient is great, repeat programming of the implant cannot be carried out because the same audiologist cannot be present in the two locations quickly enough. However, on the basis of this and other studies it seems reasonable to forego the face-to-face programming and expect that remote programming will produce equivalent maps to those generated in conventional consultations.

The system described was developed for patients with a particular implant (Med-el). Other cochlear implant manufacturers use different hardware to programme their implants. Some changes will be required to the communication protocols to enable our system to work with their cochlear implants.

Although not tested, the Silverlight application will run under other operating systems, e.g. Apple's OS X and Linux. However, as the programming software runs only in a Microsoft Windows environment, the audiologist's computer will have to use a Windows operating system.

\section{Conclusions}

The present study shows that remote programming of cochlear implants can be conducted reliably with relatively simple equipment, potentially in the homes of remotely located patients assisted by a family member. Remote programming required only a slightly longer consultation session.

\section{Acknowledgements}

We thank the cochlear implant recipients who took part in the study, ESIA Implant Centre audiologists for assistance in recruiting participants, staff at the Med-el Perth office for the loan of equipment and advice, and Craig Lowrie and Ben Upson for IT assistance. Funding for the project was received from Med-el, and the project was also supported by ESIA's Gift of Hearing.

\section{References}

1. World Health Organization. Millions of people in the world have hearing loss that can be treated or prevented. See http:// www.who.int/pbd/deafness/news/Millionslivewithhearing loss.pdf (last checked 18 February 2014).
2. Goulios H, Patuzzi RB. Audiology education and practice from an international perspective. Int $J$ Audiol 2008;47:647-64.

3. Swanepoel de W, Clark JL, Koekemoer D, et al. Telehealth in audiology: the need and potential to reach underserved communities. Int J Audiol 2010;49:195-202.

4. Campos PD, Ferrari DV. Teleaudiology: evaluation of teleconsultation efficacy for hearing aid fitting. J Soc Bras Fonoaudiol 2012;24:301-8 [English, Portuguese].

5. Swanepoel de W, Hall JW. A systematic review of telehealth applications in audiology. Telemed $J E$ Health 2010;16:181-200.

6. Franck K. Telemedicine offers remote cochlear implant programming. Volta Voices January 2006.

7. Ramos A, Rodriguez C, Martinez-Beneyto P, et al. Use of telemedicine in the remote programming of cochlear implants. Acta Otolaryngol 2009;129:533-40.

8. McElveen JT, Blackburn EL, Green JD, McLear PW, Thimsen DJ, Wilson BS. Remote programming of cochlear implants: a telecommunications model. Otol Neurotol 2010;31:1035-40.

9. Wesarg T, Wasowski A, Skarzynski H, et al. Remote fitting in Nucleus cochlear implant recipients. Acta Otolaryngol 2010;130:1379-88.

10. Hughes ML, Goehring JL, Baudhuin JL, et al. Use of telehealth for research and clinical measures in cochlear implant recipients: a validation study. J Speech Lang Hear Res 2012;55:1112-27.

11. Ling D. Speech and the Hearing-Impaired Child: Theory and Practice. Washington DC: Alexander Graham Bell Association for the Deaf, 1976.

12. Norman G. Likert scales, levels of measurement and the "laws" of statistics. Adv Health Sci Educ Theory Pract 2010; 15:625-32.

13. American Academy of Audiology. The Use of Telehealth/ Telemedicine to Provide Audiology Services. See http:// www.audiology.org/advocacy/publicpolicyresolutions/documents/telehealthresolution200806.pdf (last checked 18 February 2014).

14. Lewis C, Packman A, Onslow M, et al. A phase II trial of telehealth delivery of the Lidcombe Program of Early Stuttering Intervention. Am J Speech-Lang Pat 2008; 17:139-149.

15. Jennett PA, Affleck Hall L, Hailey D, et al. The socio-economic impact of telehealth: a systematic review. $J$ Telemed Telecare 2003;9:311-20.

16. Humes LE. Understanding the speech-understanding problems of the hearing impaired. $J$ Am Acad Audiol 1991;2:59-69.

17. Gaylor JM, Raman G, Chung M, et al. Cochlear implantation in adults: a systematic review and meta-analysis. JAMA Otolaryngol Head Neck Surg 2013;139:265-72.

18. Convery E, Keidser G, Dillon H, Hartley L. A self-fitting hearing aid: need and concept. Trends Amplif 2011;15:157-66.

19. Convery E, Keidser G, Hartley L, Caposecco A, Hickson L, Meyer C. Management of hearing aid assembly by urbandwelling hearing-impaired adults in a developed country: implications for a self-fitting hearing aid. Trends Amplif 2011;15:196-208. 